\title{
Exploring the intrinsic limit of the charge-carrier-induced increase of the Curie temperature of Lu- and La-doped EuO thin films
}

\author{
R. Held, ${ }^{1}$ T. Mairoser, ${ }^{2}$ A. Melville, ${ }^{1}$ J. A. Mundy,${ }^{3}$ M. E. Holtz, ${ }^{3}$ D. Hodash $\odot,{ }^{1}$ Z. Wang, ${ }^{1}$ J. T. Heron, ${ }^{1}$ \\ S. T. Dacek $\odot,{ }^{1}$ B. Holländer, ${ }^{4}$ D. A. Muller, ${ }^{3,5}$ and D. G. Schlom ${ }^{1,5,6}$ \\ ${ }^{1}$ Department of Materials Science and Engineering, Cornell University, Ithaca, New York 14853, USA \\ ${ }^{2}$ Zentrum für Elektronische Korrelationen und Magnetismus, Universität Augsburg, Universitätsstraße 1, D-86159 Augsburg, Germany \\ ${ }^{3}$ School of Applied and Engineering Physics, Cornell University, Ithaca, New York 14853, USA \\ ${ }^{4}$ Peter Grünberg Institut, Forschungszentrum Jülich, D-52425 Jülich, Germany \\ ${ }^{5}$ Kavli Institute at Cornell for Nanoscale Science, Ithaca, New York 14853, USA \\ ${ }^{6}$ Leibniz-Institut für Kristallzüchtung, Max-Born-Str. 2, 12489 Berlin, Germany
}

(Received 20 June 2020; accepted 28 September 2020; published 20 October 2020)

\begin{abstract}
Raising the Curie temperature $T_{\mathrm{C}}$ of the highly spin-polarized semiconductor EuO by doping it with rare-earth elements is a strategy to make EuO more technologically relevant to spintronics. The increase of $T_{\mathrm{C}}$ with free carrier density $n$ and the surprisingly low dopant activation $p$, found in Gd-doped $\mathrm{EuO}$ thin films [Mairoser et al., Phys. Rev. Lett. 105, 257206 (2010)], raised the important question of whether $T_{\mathrm{C}}$ could be considerably enhanced by increasing $p$. Using a low-temperature growth method for depositing high-quality Lu-doped EuO films we attain high dopant activation $(p)$ values of up to $67 \%$, effectively more than doubling $p$ as compared to adsorption-controlled growth of $\mathrm{Lu}$ - and $\mathrm{Gd}$-doped EuO. Relating $n, p$, and lattice compression of La- and $\mathrm{Lu}-$ doped $\mathrm{EuO}$ films grown at different temperatures to the $T_{\mathrm{C}}$ of these samples allows us to identify several different mechanisms influencing $T_{\mathrm{C}}$ and causing an experimental maximum in $T_{\mathrm{C}}$. In addition, scanning transmission electron microscopy in combination with electron energy loss spectroscopy measurements on La-doped EuO indicate that extensive dopant clustering is one, but not the sole reason for dopant deactivation in rare-earth doped EuO films.
\end{abstract}

DOI: 10.1103/PhysRevMaterials.4.104412

\section{INTRODUCTION}

Many of the remarkable properties of the semiconductor $\mathrm{EuO}$, including strong ferromagnetism, large Faraday rotation, and a giant insulator-to-metal transition, were discovered more than 40 years ago [1-4]. More recent work on thin films has demonstrated $>90 \%$ spin polarization of electrical currents in doped $\mathrm{EuO}$ and the successful epitaxial integration of EuO with silicon, GaAs, and $\mathrm{GaN}$ [5-7]. The increasing structural perfection of EuO films grown directly on silicon may allow fabricating efficient spin filter contacts to silicon providing an alternative route for integrating spin filter functionality into silicon [8-10]. These qualities render EuO an outstanding material for spintronic studies and proof-ofconcept devices. Furthermore, deposition of the easily overoxidized half-metallic semiconductor EuO does not always require expensive ultrahigh-vacuum equipment: By employing topotactic transformation $\mathrm{EuO}$ thin films with excellent quality can be fabricated using just high-vacuum means [11].

Stoichiometric EuO has a Curie temperature $\left(T_{\mathrm{C}}\right)$ of $69 \mathrm{~K}$ [12], which is comparable to the Curie temperatures of other

Published by the American Physical Society under the terms of the Creative Commons Attribution 4.0 International license. Further distribution of this work must maintain attribution to the author(s) and the published article's title, journal citation, and DOI. spintronic materials like standard dilute semiconductor systems, such as manganese-doped GaAs, and well below the $T_{\mathrm{C}}$ values needed for commercial devices, but by doping $\mathrm{EuO}$ with oxygen vacancies $\left(\mathrm{EuO}_{1-x}\right)$ [4] or with rare-earth atoms such as gadolinium, lanthanum, lutetium, and scandium [13-18], the Curie temperature can be considerably enhanced. In addition, compressive epitaxial strain is expected to further increase $T_{\mathrm{C}}[19,20]$.

Below $T_{\mathrm{C}}$, charge carrier transfer occurs in $\mathrm{EuO}$ from the donor level into the lower part of the Zeeman-split spin-polarized EuO $5 d-6 s$ conduction band [5]. One of the mechanisms responsible for the $T_{\mathrm{C}}$ increase by doping is the enhancement of the indirect exchange between the ferromagnetically ordered $\mathrm{Eu} 4 f$ moments mediated by these additional free charge carriers up to a critical carrier density $n_{\mathrm{C}}$. According to theoretical models describing this scenario, carrier densities beyond $n_{\mathrm{C}}$ lead to magnetic instabilities [21] or antiferromagnetic ordering [19] and to a $T_{\mathrm{C}}$ reduction.

Importantly, not all dopant atoms contribute an electron to the conduction band. The only published measurements quantifying the relationship between the doping concentration $x$ and free carrier density $n$ (allowing calculation of the fractions of active and inactive dopants), and $T_{\mathrm{C}}$ of doped $\mathrm{EuO}$, are on Gd-doped EuO films [22-24]. These experiments show a roughly logarithmic increase of $T_{\mathrm{C}}$ up to $129 \mathrm{~K}$ when plotted as a function of $n$. For high doping concentrations $x \gtrsim 10 \%$ the mobile carrier density saturates around 
$1 \times 10^{21} \mathrm{~cm}^{-3}$. But most importantly, these measurements reveal a surprisingly low Gd dopant activation $p$ of less than $35 \%$ for all films. Later, it was shown that $T_{\mathrm{C}}$ and $p$ in Gd-doped $\mathrm{EuO}$ decrease strongly with increasing deposition temperature above $T \sim 350^{\circ} \mathrm{C}$ [24]. Interestingly, all of the samples in these works show high structural quality in either low-energy electron diffraction or x-ray diffraction (XRD) measurements with narrow rocking curve full width at half maximum (FWHM) values around $0.01^{\circ}$ and no indications of possible structural changes of the films caused by Gd doping.

These prior findings raise several questions the answers of which could have tremendous implications on potential applications of EuO. First, which mechanisms are responsible for the deactivation of dopant carriers in EuO? Second, is it possible to increase the dopant activation and Curie temperature by optimizing the growth conditions or by using different dopants? Third, what are the experimental limits to the Curie temperature given by the various doping methods and strain?

Using a low-temperature, close to flux-matched growth method, we reach higher dopant activations and higher carrier densities than prior studies in Lu-doped $\mathrm{EuO}$ thin films, effectively tripling these values in samples with high $T_{\mathrm{C}}$ as compared to samples grown using standard adsorptioncontrolled growth. By comparing $n, p$, and $T_{\mathrm{C}}$ of La- and Lu-doped $\mathrm{EuO}$ films grown at different temperatures, we show that increasing $n$ beyond $\sim 2-4 \times 10^{20} \mathrm{~cm}^{-3}$ has only a very limited effect on $T_{\mathrm{C}}$ in these samples. We find indications that at these high carrier densities, the maximum $T_{\mathrm{C}}$ of La- and Lu-doped samples is instead determined by a superposition of effects from dopant ion size, concomitant lattice compression, and reduced direct exchange caused by high doping concentrations of nonmagnetic ions. This is corroborated by $\mathrm{x}$-ray diffraction and STEM-EELS measurements of La-doped EuO films that also rule out extended crystallographic defects as reason for the limited $T_{\mathrm{C}}$ of these films.

\section{METHODS}

To perform these experiments we deposited singlecrystalline epitaxial La- and Lu-doped $\mathrm{EuO}$ thin films on (110) $\mathrm{YAlO}_{3}$ and (110) $\mathrm{LuAlO}_{3}$ substrates by reactive oxide molecular-beam epitaxy using two different growth techniques: standard adsorption-controlled growth and lowertemperature, close to flux-matched deposition. The thickness of the films was chosen to be $\sim 35 \mathrm{~nm}$, which is sufficiently thin to avoid structural relaxation of the $\mathrm{EuO}$ films grown on the $\mathrm{YAlO}_{3}$ and $\mathrm{LuAlO}_{3}$ substrates $[20,25]$. To dope the EuO films, we evaporated $\mathrm{Eu}$ and the dopant element at the same time from separate effusion cells at the desired ratio using a quartz crystal microbalance to calibrate the fluxes of the separate molecular beams. After growth, we capped the samples in situ with $50 \mathrm{~nm}$ of amorphous $\mathrm{LaAlO}_{3}$ or $30 \mathrm{~nm}$ of amorphous silicon to protect them from further oxidation when exposed to air. We investigated the crystalline quality of almost all of the 42 films that are the subject of this study by four-circle x-ray diffraction.

It is challenging to grow stoichiometric $\mathrm{EuO}$ thin films, mainly because of their tendency to form higher oxides such as $\mathrm{Eu}_{3} \mathrm{O}_{4}$ and $\mathrm{Eu}_{2} \mathrm{O}_{3}$. This problem could be solved for many applications by using adsorption-controlled growth at substrate temperatures $>400^{\circ} \mathrm{C}$. Previous work showed that using this method it is possible to reproducibly grow close-to-stoichiometric $\mathrm{EuO}$ films with excellent crystalline quality according to XRD [20,23-26]. Reaching a similar film quality with lower-temperature growth is much more challenging.

At the lower substrate temperatures of $T=$ $250{ }^{\circ} \mathrm{C}-275^{\circ} \mathrm{C}$ used for deposition of the close to flux-matched films in this study, almost all of the impinging $\mathrm{Eu}$ atoms stick to the growing film surface. This leads to a considerably smaller growth window for stoichiometric $\mathrm{EuO}$ as compared to growth at higher substrate temperature. As oxygen vacancies in $\mathrm{EuO}$ are expected to increase $n$ and can also lead to a considerable $T_{\mathrm{C}}$ increase, $[4,18]$ it is imperative to avoid these defects to be able to determine the intrinsic effect of rare-earth doping. On the other hand, oxygen-rich deposition conditions that lead to the formation of $\mathrm{Eu}_{3} \mathrm{O}_{4}$ or $\mathrm{Eu}_{2} \mathrm{O}_{3}$ are expected to reduce $n$ and $T_{\mathrm{C}}$. We overcame these conflicting issues by precisely mapping the growth window as a function of substrate temperature and oxygen partial pressure and meticulous calibration of the deposition conditions utilizing a residual gas analyzer (RGA) for accurate control of the relative oxygen partial pressure and reflection high-energy electron diffraction (RHEED) for in situ quality control. More details on film growth methods can be found in the Supplemental Material [27]. The adsorption-controlled samples were grown at $T=400{ }^{\circ} \mathrm{C}$ according to the recipe described elsewhere [24-26].

The $T_{\mathrm{C}}$ of the films was determined using superconducting quantum interference device (SQUID) magnetometry or vibrating sample magnetometry (VSM) following Ref. [28]. Measured magnetization characteristics are consistent with corresponding curves found in the literature (see Supplemental Material [27]). We determined the in-plane saturation magnetization $\left(M_{\text {sat }}\right)$ and field $\left(H_{\text {sat }}\right)$ from hysteresis loops measured at $5 \mathrm{~K}$.

We measured the Hall resistance $R_{\mathrm{H}}$ at $T=5 \mathrm{~K}$ using $\sim 100-\mu \mathrm{m}$-wide Hall bars patterned by photolithography in combination with in situ ion etching and sputter deposition following the procedure described in Ref. [22] (see Supplemental Material [27]). These Hall effect measurements were used to determine the mobile charge carrier density $n$ in the La- and Lu-doped EuO films. The ratio of $n$ to the concentration of rare-earth dopants determined $p$, the dopant activation.

\section{RESULTS}

Figure 1(a) compares $T_{\mathrm{C}}$ and $n$ of Lu-doped EuO films grown using adsorption-controlled or close to flux-matched conditions on $\mathrm{LuAlO}_{3}$. It is evident that the samples deposited with both techniques reach comparable maximum $T_{\mathrm{C}}$ values around $126 \mathrm{~K}$, but the Lu-doped samples grown at lower $T$ exhibit much higher maximum mobile carrier densities as compared to their counterparts grown by adsorption control. The samples grown at $T=250^{\circ} \mathrm{C}$ reach carrier densities of $\sim 1.6 \times 10^{21} \mathrm{~cm}^{-3}$ at $8 \%-12 \%$ doping concentration, which is 3.4 times the maximum value of the samples grown at $T=$ $400{ }^{\circ} \mathrm{C}$. This difference also manifests in the dopant activation [Fig. 1(b)]. While the Lu-doped samples grown at $T=400^{\circ} \mathrm{C}$ exhibit activation levels below 33\%, the Lu-doped samples 

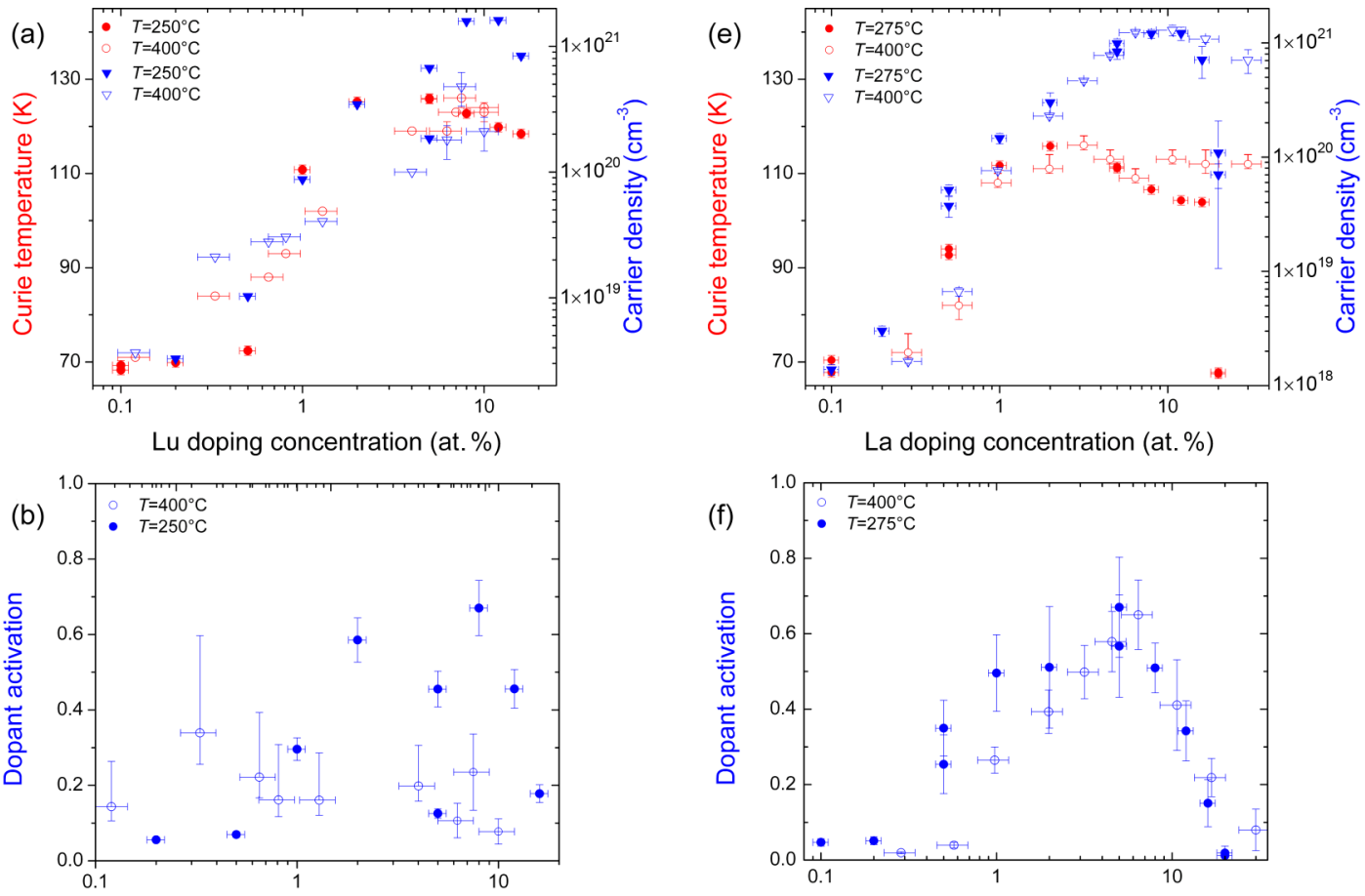

Lu doping concentration (at. \%)

La doping concentration (at. \%)
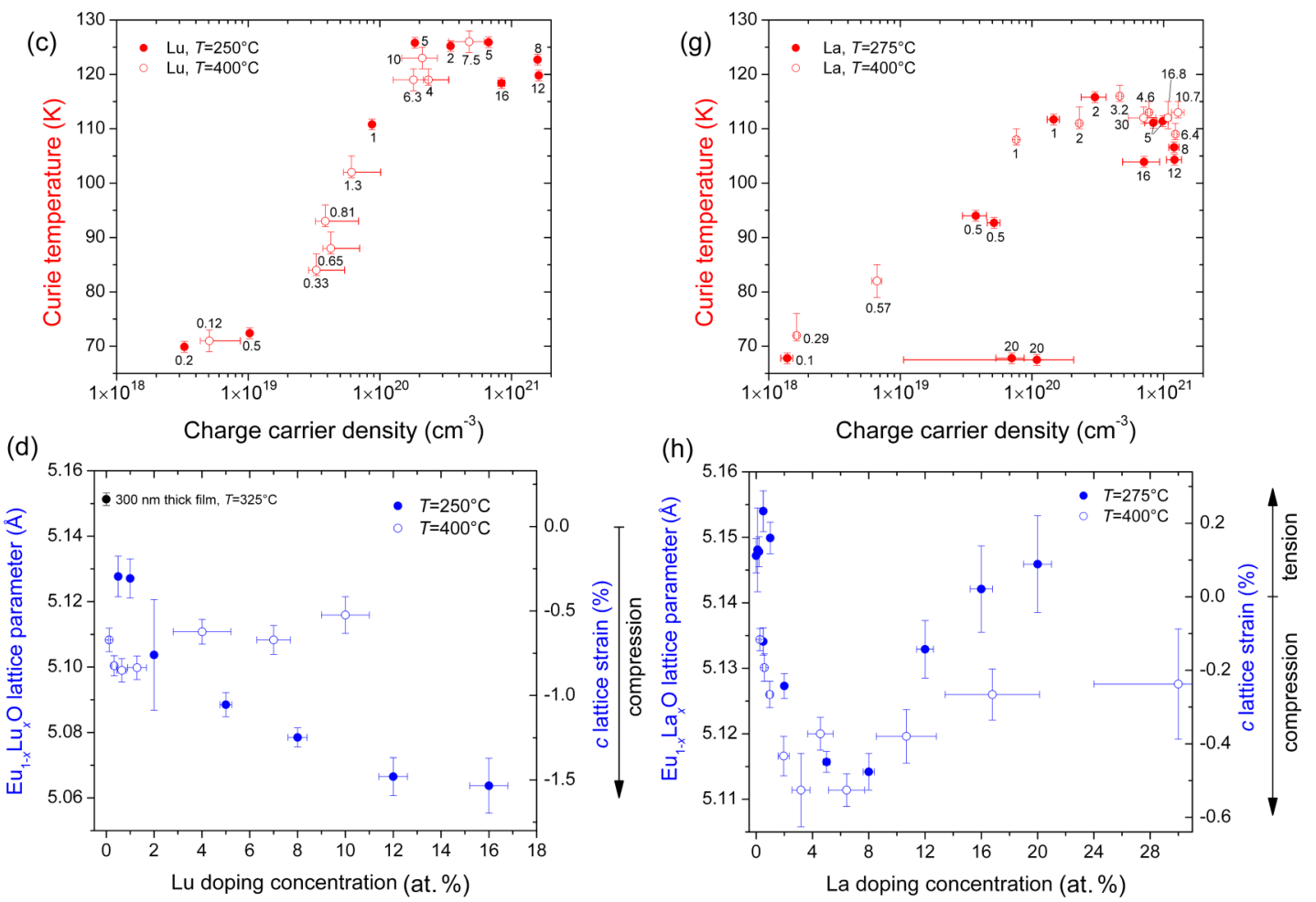

FIG. 1. (a) Dependence of $T_{\mathrm{C}}$ and $n$ on $x$ in $\mathrm{Eu}_{1-x} \mathrm{Lu}_{x} \mathrm{O}$ films grown at different substrate temperatures $T$ on $\mathrm{LuAlO}_{3}$ and measured at $5 \mathrm{~K}$; (b) calculated dopant activation $p$ as a function of the doping concentration in atomic percent in $\mathrm{Eu}_{1-x} \mathrm{Lu}_{x} \mathrm{O}$ for the same samples. (c) Dependence of $T_{\mathrm{C}}$ on $n$ in $\mathrm{Eu}_{1-x} \mathrm{Lu}_{x} \mathrm{O}$ films grown at different substrate temperatures $T$ measured at $5 \mathrm{~K}$. The numbers at the data points denote the doping concentrations in atomic percent. (d) $\mathrm{Eu}_{1-x} \mathrm{Lu}_{x} \mathrm{O}$ out-of-plane lattice spacing $c$ and calculated $c$ lattice strain as compared to bulk $\mathrm{EuO}$ as functions of $x$, derived from the 002 peak positions in $\theta-2 \theta$ scans. The full symbols represent data points of samples grown at $T=250^{\circ} \mathrm{C}$ and the empty symbols represent data points of samples grown at $T=400^{\circ} \mathrm{C}$. For comparison, the measured $c$ axis of a 300-nm-thick undoped $\mathrm{EuO}$ film grown on $\mathrm{LuAlO}_{3}$ at $T=325^{\circ} \mathrm{C}$ is included. At this thickness, this film is expected to be relaxed [20]. (e)-(h) show the same measurements and calculation results as (a)-(d) but with $\mathrm{Eu}_{1-x} \mathrm{La}_{x} \mathrm{O}$ films. Please note that the lower growth temperature of these samples was $T=275^{\circ} \mathrm{C}$. 
grown at $T=250{ }^{\circ} \mathrm{C}$ reach a maximum activation of up to $67 \%$. In the doping regime where high- $T_{\mathrm{C}}$ values are reached the difference is even more pronounced. Not all of the lowtemperature grown samples exhibit high carrier densities and dopant activations, suggesting a strong influence of growth details on these properties. It is worth noting that the low dopant activation of Lu-doped samples grown at $T=400{ }^{\circ} \mathrm{C}$ is consistent with the low dopant activation previously reported for Gd-doped samples grown in the adsorption-controlled growth regime [22-24].

The data in Fig. 1(c) also show that the $T_{\mathrm{C}}$ of all samples intially strongly increases with increasing $n$ above a threshold of $\sim 5-10 \times 10^{18} \mathrm{~cm}^{-3}$. Up to $n \sim 2 \times 10^{20} \mathrm{~cm}^{-3}$ samples with higher $n$ typically also exhibit the higher $T_{\mathrm{C}}$. This trend continues for the samples grown at $T=400{ }^{\circ} \mathrm{C}$ up to the highest $n$ values that are reached $\left(4.7 \times 10^{20} \mathrm{~cm}^{-3}\right)$. It becomes clear, however, from the samples grown at lower temperature that reach higher carrier densities, that $T_{\mathrm{C}}$ ultimately levels out at $\sim 126 \mathrm{~K}, n=2-5 \times 10^{20} \mathrm{~cm}^{-3}$, and $2 \%-8 \%$ doping concentration and does not reach higher values, even for much higher $n$ (up to $\sim 1.6 \times 10^{21} \mathrm{~cm}^{-3}$ ). For higher doping concentrations $T_{\mathrm{C}}$ is reduced, despite the very high $n \sim 8 \times 10^{20} \mathrm{~cm}^{-3}$ of the samples grown at $T=250^{\circ} \mathrm{C}$.

Therefore, increasing $n$ beyond $\sim 2 \times 10^{20} \mathrm{~cm}^{-3}$ does not lead to higher $T_{\mathrm{C}}$ values of these films, as can be inferred from the overall trend and exemplified at the two 5\%-doped samples grown at $T=250^{\circ} \mathrm{C}$ that have strongly differing $n$ $\left(1.9 \times 10^{20} \mathrm{~cm}^{-3}\right.$ vs $\left.6.7 \times 10^{20} \mathrm{~cm}^{-3}\right)$, but exactly the same $T_{\mathrm{C}}$ of $126 \mathrm{~K}$.

What other effects could play a role for the observed maximum in $T_{\mathrm{C}}$ ? By analyzing the 002 peak positions in $\theta-2 \theta$ scans of the Lu-doped samples grown at $T=250{ }^{\circ} \mathrm{C}$ on $\mathrm{LuAlO}_{3}$ [Fig. 1(d)] we found an increasing $c$ lattice compression (the out-of-plane direction) of up to $-1.6 \%$ for the samples grown at $T=250{ }^{\circ} \mathrm{C}$ as a function of increasing doping concentration. For the EuO bulk lattice parameter we used $a=5.1439 \AA$ [29]. The lattice compression of the samples grown by adsorption control shows a different behavior and is almost constant around $-0.7 \%$. Interestingly, despite this difference in $c$-axis strain, the maximum $T_{\mathrm{C}}$ of the samples is the same and the $T_{\mathrm{C}}$ of the samples grown at $T=250{ }^{\circ} \mathrm{C}$ is already close to the maximum value for $2 \%-5 \%$ doping concentration, although the lattice compression is still increasing for higher $x$.

To investigate possible effects of dopant ion size we also measured $T_{\mathrm{C}}$ and $n$ of two series of La-doped EuO films deposited on $\mathrm{YAlO}_{3}$, one grown at $T=400{ }^{\circ} \mathrm{C}$ using standard adsorption-controlled growth and one grown close to the fluxmatched regime, at $T=275^{\circ} \mathrm{C}$ [Fig. 1(e)]. When substituted for $\mathrm{Eu}^{2+}$ in the EuO lattice, lanthanum is expected to be in a $3+$ valence state just like lutetium, so that it should also donate one electron per ion. With an ionic radius of $1.03 \AA$ it is, however, about $20 \%$ larger than the $\mathrm{Lu}^{3+}$ ion and closer to the size of $\mathrm{Eu}^{2+}$ (1.17 $\AA$ ) [30].

Remarkably, the La-doped samples reach high charge carrier densities of up to $1.4 \times 10^{21} \mathrm{~cm}^{-3}$, independent of the growth temperature-but the maximum $T_{\mathrm{C}}$ of both sample batches is $\sim 114 \mathrm{~K}$, about $12 \mathrm{~K}$ lower than the maximum $T_{\mathrm{C}}$ of the Lu-doped samples. The peak in $T_{\mathrm{C}}$ is reached at $2 \%-4 \%$ doping concentration, far before the peak in $n$ is reached at
10\%-12\% doping concentration. These effects clearly show that in La-doped as well as Lu-doped EuO high $n$ values are insufficient for reaching high maximum $T_{\mathrm{C}}$ values. At doping concentrations $>4 \%$, the $T_{\mathrm{C}}$ of the low-temperature grown La-doped samples is reduced by similar amounts as the low-temperature grown Lu-doped samples, whereas the $T_{\mathrm{C}}$ of the samples grown by adsorption control is reduced by smaller amounts.

The maximum dopant activation of the two La-doped sample batches grown at different temperatures is comparable [Fig. 1(f)]. The dopant activation of the samples grown at lower temperature is only higher for doping concentrations $\sim 0.5 \%-2 \%$. Therefore, growth temperature plays a much less important role for the maximum $n$ in La-doped EuO as compared to Lu-doped EuO.

When plotted as a function of $n$ [Fig. 1(g)], the $T_{\mathrm{C}}$ of the La-doped samples initialy shows a strong increase above a threshold of $n \sim 2-7 \times 10^{18} \mathrm{~cm}^{-3}$ and a saturation for $n \gtrsim 3-5 \times 10^{20} \mathrm{~cm}^{-3}$, similar to the Lu-doped samples. In this $n$ range, samples with higher $n$ also typically exhibit the higher $T_{\mathrm{C}}$, just like the Lu-doped samples. An exception is very highly La-doped samples $(20 \%)$ grown at $T=275^{\circ} \mathrm{C}$ that show enhanced carrier densities, but low $T_{\mathrm{C}}$ values of $\sim 68 \mathrm{~K}$. The RHEED patterns of these samples changed during growth indicating that a structural change of the films due to the high doping is likely responsible for the low $T_{\mathrm{C}}$. At high $n$ and high $x, T_{\mathrm{C}}$ is reduced - an effect that is more pronounced for the samples grown at lower temperature and is also found in Lu-doped samples [Fig. 1(c)].

The reduction of $T_{\mathrm{C}}$ at doping levels higher than $2 \%-8 \%$ for $\mathrm{Lu}$ and $2 \%-4 \%$ for La could be rooted in the weakening of the direct exchange between $\mathrm{Eu}^{2+}$ ions caused by the disturbance of the magnetic lattice as expected in case of doping with nonmagnetic ions. The larger lattice compression found for Lu-doped samples as compared with La-doped samples grown at low substrate temperature (see below) may partially alleviate this effect so that $T_{\mathrm{C}}$ is not reduced before reaching higher $x$ values. It should be noted that for $\mathrm{Gd}$ doping, $T_{\mathrm{C}}$ has been found to increase up to higher concentrations (at least $10 \%$ ) [22], as may be expected due to the magnetism of the $\mathrm{Gd}^{3+}$ ion. The same effect could be responsible for the slightly higher maximum $T_{\mathrm{C}}$ found for $\mathrm{Gd}$-doped samples (129 K, [22]) as compared to Lu-doped samples (126 K) and the less pronounced drop in $T_{\mathrm{C}}$ for high $x$ in these samples as compared to Lu- and La-doped samples.

Analysis of the $c$ lattice spacing reveals that the lattice compression caused by $\mathrm{La}$ doping of $\mathrm{EuO}$ films grown at $T=275^{\circ} \mathrm{C}$ and $T=400{ }^{\circ} \mathrm{C}$ on $\mathrm{YAlO}_{3}$ reaches a maximum of $-0.6 \%$ around $6 \%$ doping [Fig. 1(h)]. This maximal compressive strain is considerably smaller than the compression of Lu-doped films grown at $T=250{ }^{\circ} \mathrm{C}$ on $\mathrm{LuAlO}_{3}(-1.6 \%$ at $16 \%$ doping). Due to the $20 \%$ larger $\mathrm{La}$ ion size this direction of this effect is expected; however, the magnitude is surprisingly large. At high doping concentrations $>8 \%$ the lattice compression of all the La-doped films is reduced, whereas the lattice of the low-temperature grown Lu-doped samples gets increasingly compressed up to the highest investigated concentration of $16 \%$. It is not yet clear what causes this effect (for possible reasons see the Supplemental Material [27]). 


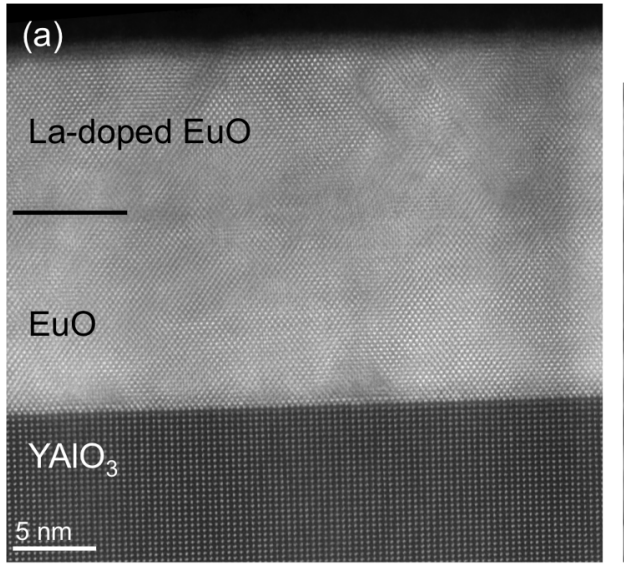

(b) $L a-M_{4,5}$

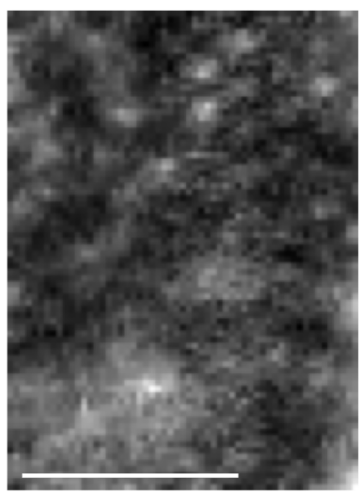

(c) Eu- $M_{4,5}$

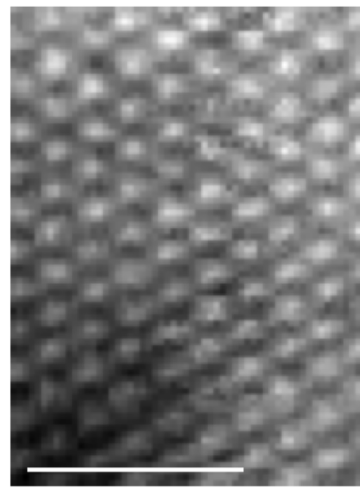

(d) Combined

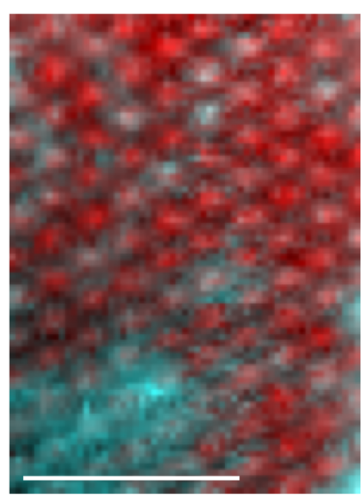

(e) La- $N_{4,5}$, Eu- $N_{4,5}$

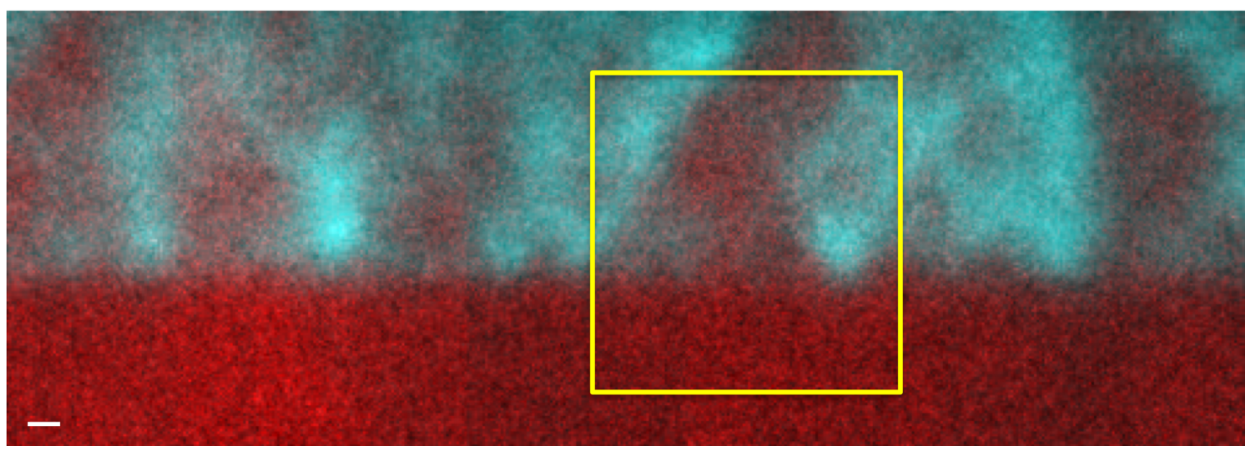

(f) $\mathrm{La}-M_{4,5}$, Eu- $M_{4,5}$

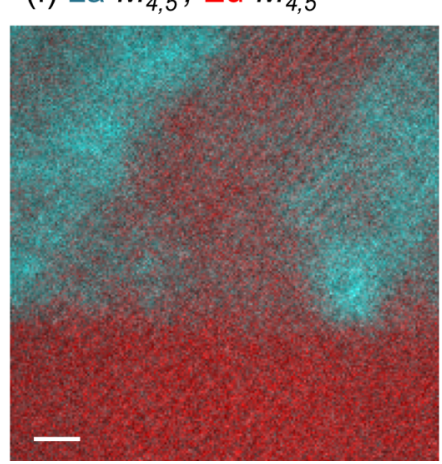

FIG. 2. Spectroscopic imaging of a $\mathrm{La}_{0.08} \mathrm{Eu}_{0.92} \mathrm{O}$ film grown on an undoped EuO buffer layer on a $\mathrm{YAlO}_{3}$ substrate in the adsorptioncontrolled growth regime. (a) HAADF-STEM image of the sample. Atomic-resolution spectroscopic images of the $\mathrm{La}_{0.08} \mathrm{Eu}_{0.92} \mathrm{O}$ region using the signal from the La- $M_{4,5}$ and Eu- $M_{4,5}$ edges are shown in (b) and (c), respectively. (d) The combined spectroscopic image with lanthanum in turquoise and europium in red shows that the lanthanum signal is inhomogeneous, indicating clustering of lanthanum atoms. (e) A lowmagnification EELS spectroscopic image using the La- $N_{4,5}$ and Eu- $N_{4,5}$ edges demonstrates that the lanthanum preferentially clusters the near [010] and [100] zone axes. (f) A high-resolution image of a selected region from (e). Scale bars in (b)-(f) are $1 \mathrm{~nm}$.

Altendorf previously found an approximately linear $c$ axis lattice contraction with increasing doping concentration in XRD measurements of Sc-doped EuO [17]. The ionic radius of $\mathrm{Eu}^{2+}$ is $1.17 \AA$, as compared to $0.86 \AA$ for $\mathrm{Lu}^{3+}, 1.03 \AA$ for $\mathrm{La}^{3+}$, and $0.75 \AA$ for Sc [30]. As $\mathrm{La}^{3+}$, $\mathrm{Lu}^{3+}$, and $\mathrm{Sc}^{3+}$ are chemically very similar, the smaller $\mathrm{Lu}^{3+}$ and $\mathrm{Sc}^{3+}$ ion sizes and concomitant stronger lattice compression should also contribute to the higher $T_{\mathrm{C}}$ found in these samples.

Dopant deactivation in conventional semiconductors such as silicon is an issue that has been studied with a large variety of techniques in a vast amount of literature (see, e.g., Ref. [31] and references therein). One of the most important effects leading to the deactivation of dopant carriers is defects in the crystallographic microstructure, the formation of which can depend on film growth conditions, doping level, and the individual dopant/host combination. Therefore, the interdependencies of doping level, density of free carriers, and Curie temperature ideally should also be related to film microstructure when studying dopant deactivation in EuO.

High-angle annular dark field STEM (HAADF STEM) has previously been used to identify individual dopant atoms, e.g., antimony dopant location and clustering in highly doped silicon $\left(n=9.35 \times 10^{20} \mathrm{~cm}^{-3}\right)$ [32]. In that case, the high atomic number contrast between the antimony $(Z=51)$ and the host silicon $(Z=14)$ led to a large contrast in HAADF STEM; here the dopant atoms (La, $Z=57$ and $\mathrm{Lu}, Z=71$ ) have an atomic number similar to europium $(Z=63)$, which makes it harder to locate individual dopant atoms. We thus use two-dimensional (2D) EELS spectroscopic imaging to determine the dopant positions.

Indeed the combination of HAADF STEM and EELS has been successfully used to discern valence changes of the EuO at the $\mathrm{EuO} / \mathrm{Si}$ interface indicating the formation of impurity phases [33]. Here we use the same techniques to investigate the dopant distribution and dopant cluster formation in La-doped EuO films. We focus on lanthanum doping as the La- $N_{4,5}$ and La- $M_{4,5}$ EELS edges used precede the corresponding Eu- $N_{4,5}$ and Eu- $M_{4,5}$ edges; in contrast, the Lu- $N_{4,5}$ and $\mathrm{Lu}-M_{4,5}$ edges sit on the background of the corresponding europium edges making detection of small dopant quantities more difficult. Doping concentrations were between $5 \%$ and $8 \%$, as the maximum in $T_{\mathrm{C}}$ for the La-doped samples occurs in this doping range. The La- $M_{4,5}$ and Eu- $M_{4,5}$ edges were used to probe the atomic-scale clustering and the La- $N_{4,5}$ and $\mathrm{Eu}-N_{4,5}$ edges to acquire images with a wide field of view at lower spatial resolution.

A HAADF STEM image of an $8 \%$ La-doped EuO film grown in the adsorption-controlled regime is shown in Fig. 2(a) (see the Supplemental Material [27] for more 

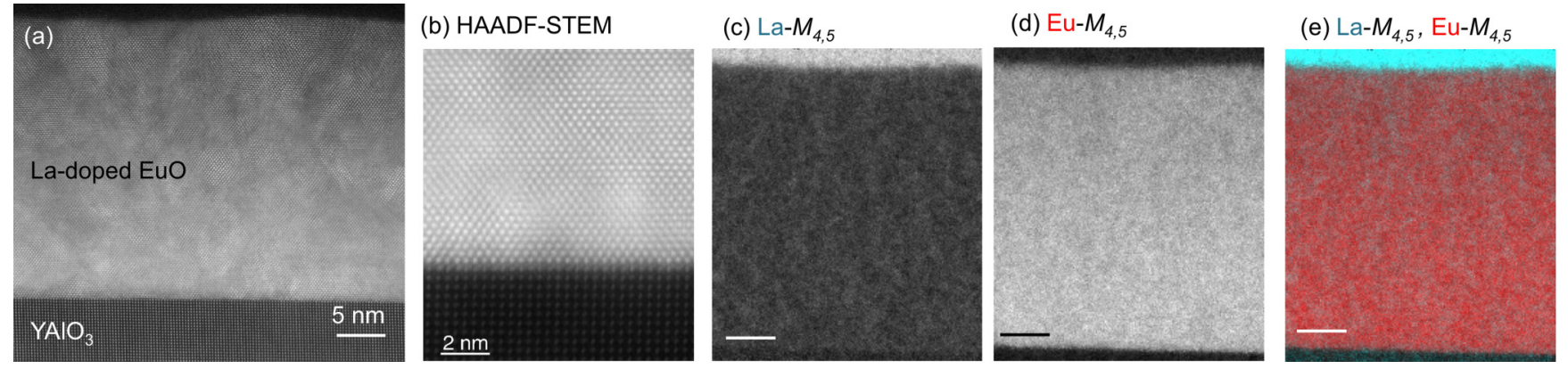

FIG. 3. STEM EELS of an $8 \%$ La-doped EuO film grown close to the flux-matching regime at a relatively low substrate temperature of $T=275^{\circ} \mathrm{C}$. (a) Overview image of the sample. The top $12 \mathrm{~nm}$ of the EuO film has been oxidized (see Supplemental Material [27]). (b) Atomic-resolution HAADF-STEM image, demonstrating that the interface between the film and the substrate lacks impurity phases. Chemical maps of the lanthanum (c) and europium (d) show homogeneous distribution of these elements. (e) shows a combined color map of the film with lanthanum in turquoise and europium in red. The La content of the $\mathrm{LaAlO}_{3}$ capping layer (bright turquoise) is $20 \%$. Scale bars in (c)-(e) are $5 \mathrm{~nm}$.

information). In Figs. 2(b)-2(d), we show atomic-resolution images of the lanthanum and europium concentrations; even in this small field of view, there is significant fluctuation in the lanthanum concentration ranging from atomic columns with nearly full occupancy of europium to those with nearly $20 \%$ lanthanum. This variation was observed over larger fields of view at both atomic and low resolution in Figs. 2(d) and 2(f), respectively. There we note that not only do the lanthanum atoms cluster, they do so along preferred [100] and [010] zone axes. Note that signs of this dopant clustering are not evident in $\theta-2 \theta$ scans or rocking curve XRD measurements.

While the exact nature and electronic structure of the clusters in La-doped $\mathrm{EuO}$ remain to be investigated, the extended dopant clustering indicates that such defects could play an important role in dopant deactivation, at least in the doped $\mathrm{EuO}$ films grown by adsorption control that tend to exhibit lower dopant activation values. We note that in silicon, pronounced dopant clustering is considered to be one of the main reasons for dopant deactivation at high dopant concentrations $n \sim 1 \times 10^{21} \mathrm{~cm}^{-3}$ [34].

Dopant clustering could also explain the smaller $T_{\mathrm{C}}$ reduction found in samples grown by adsorption control as compared to low- $T$ grown samples at high La doping levels [Fig. 1(e)]. Even at high doping levels there are regions in the samples grown by adsorption control that contain lower La concentrations (where $T_{\mathrm{C}}$ is maximal), resulting in these high, but inhomogeneously doped samples exhibiting high $T_{\mathrm{C}}$ (Fig. 2). In contrast to these samples, the lower- $T$ grown samples exhibit an almost homogeneous dopant distribution (described below), which obviously leads to a $T_{\mathrm{C}}$ reduction at high La doping levels. Finally, clustering may also influence the lattice parameters of the films and could explain the smaller $c$-axis lattice compression observed in samples grown by adsorption control.

In Fig. 3 we present an STEM EELS image of an 8\% Ladoped EuO film grown at $T=275^{\circ} \mathrm{C}$. As shown in Fig. 3(a) and in higher resolution in Fig. 3(b), the film is crystalline and the region between the film and substrate is free of impurities. In contrast to the film grown by adsorption control, the EELS spectroscopic images of the lanthanum and europium edges in Figs. 3(c)-3(e) show a homogeneous lanthanum concentration without significant dopant clustering. The dopant activation in this sample is much higher than the activation typically found in Gd-doped films, but at $p=58 \%$ it is still far from complete.

The finding of extended dopant clustering in La-doped $\mathrm{EuO}$ films grown with the growth technique that is most commonly used today-adsorption control—could be part of the explanation of the very low dopant activation values typically found in some doped $\mathrm{EuO}$ samples. Using lower growth temperatures we were able to avoid the formation of these defects, but the dopant activation still saturated at values below $80 \%$, which is insignificantly higher than the dopant activation values of the best La-doped samples grown by adsorption control. Obviously, there must be other mechanisms leading to carrier deactivation that we cannot detect in our STEM-EELS studies.

In this work we have also shown that only low-temperature, close to flux-matched growth allows achieving high lutetium activation. Thermally activated defect formation therefore suggests itself as reason for the difference in carrier deactivation in these Lu-doped films. As lanthanum and lutetium are chemically very similar, but the ionic radius of $\mathrm{Lu}^{3+}(0.86 \AA)$ is much smaller than that of $\mathrm{La}^{3+}(1.03 \AA$ ) [30], we conclude that small ion size enhances growth-temperature-related deactivation effects in rare-earth doped $\mathrm{EuO}$. This is consistent with the data on $\mathrm{Gd}$-doped EuO published by Mairoser $e t$ al. [24] who also found a strong reduction of dopant activation with increasing growth temperature. $\mathrm{As} \mathrm{Gd}^{3+}(0.938 \AA$, [30]) is also smaller than $\mathrm{La}^{3+}$, we postulate that the same effect as in the case of $\mathrm{Lu}$ doping is responsible for the low activation of Gd reported by these authors [22,24].

What kinds of defects may lead to these effects? One possibility is vacancies on the Eu site, because rather than two lanthanum dopants giving rise to two mobile electrons $\left(2 \mathrm{La}_{\mathrm{Eu}}+2 e^{\prime}\right)$ the free electrons can instead be fully compensated by the formation of a vacancy on the $\mathrm{Eu}$ site $\left(2 \mathrm{La}_{\mathrm{Eu}}+V_{\mathrm{Eu}}^{\prime \prime}\right)$. Such vacancies therefore reduce the number of free electrons. The formation of such defects would be expected to be more pronounced at higher growth temperatures, at which more Eu atoms have enough kinetic energy to leave the growing crystal surface. Smaller dopant ion size would probably strengthen this effect by lowering the formation energy of such defects, because the required EuO lattice 
distortions would be smaller. Both of these effects would be consistent with our findings.

Consequently, low deposition temperatures and avoiding $\mathrm{O}_{2}$ excess is expected to reduce the number of these defects, which should increase the density of free carriers. This effect therefore could explain the very high dopant activation values found in our low-temperature grown samples. It should be observable especially at low doping levels where even a small number of free carrier deactivating defects will lead to a strong reduction or even complete inactivation of free carriers. Indeed, Averyanov et al. [35] found recently that using relatively low deposition temperatures $\left(T \sim 430^{\circ} \mathrm{C}\right)$ and careful calibration of europium and $\mathrm{O}_{2}$ fluxes allowed generating significant free carrier densities in Gd-doped $\mathrm{EuO}$ films already at very low doping levels $<0.05 \%$.

Other effects, which are known from conventional semiconductors, such as donor deactivation distortions of the host lattice, could also play a role here [34]. A theoretical analysis of the energies of formation of different defect states and their effect on dopant deactivation could be highly useful to identify the mechanisms responsible for the dopant deactivation in EuO.

\section{CONCLUSIONS}

What conclusions can we draw from these results for the quest of reaching higher Curie temperatures of EuO by rare-earth doping? Our electrical and magnetic measurements reveal that increasing the carrier density in La- and Lu-doped samples does not always result in higher maximum $T_{\mathrm{C}}$ values. We were able to triple the maximum dopant activation in Lu-doped $\mathrm{EuO}$ thin films in the regime of high dopant concentrations and Curie temperatures by our low-temperature growth method. This led to unprecedented high carrier densities of up to $1.6 \times 10^{21} \mathrm{~cm}^{-3}$. Unfortunately, increases in $n$ did not result in higher $T_{\mathrm{C}}$. From the data we conclude that carrier densities in the wide range between $2 \times 10^{20} \mathrm{~cm}^{-3}$ and $1.6 \times 10^{21} \mathrm{~cm}^{-3}$ neither lead to enhanced nor significantly reduced $T_{\mathrm{C}}$ in these samples. In this range neither $n$ nor $T_{\mathrm{C}}$ depends on $n$ or other effects compensating the effect of $n$ on $T_{\mathrm{C}}$.

Despite high dopant activation and comparable $n$, Ladoped samples exhibit maximum $T_{\mathrm{C}}$ values of only $\sim 114 \mathrm{~K}$, about $12 \mathrm{~K}$ lower than Lu-doped samples grown at $250{ }^{\circ} \mathrm{C}$. $\mathrm{La}^{3+}$ and $\mathrm{Lu}^{3+}$ ions are both nonmagnetic, so the reason for the $T_{\mathrm{C}}$ difference cannot be due to differences of the disturbance of the magnetic lattice in EuO. Additionally, in combined STEM-EELS measurements we did not find any extended defects that could be responsible for the lower $T_{\mathrm{C}}$ of the low-temperature grown La-doped samples. Further, the sample quality according to XRD is comparable.

Lattice compression is expected to have a considerable influence on $T_{\mathrm{C}}[19]$ and a comparison of $\mathrm{x}$-ray absorption spectroscopy (XAS) measurements with band-structure calculations led to the suggestion that lattice compression underlies the high $T_{\mathrm{C}}$ of $\mathrm{Gd}$-doped $\mathrm{EuO}$ [36]. The difference in the $c$-axis contraction between La- and Lu-doped EuO that we measured by XRD is only $-0.2 \%$ for the high- $T_{\mathrm{C}}$ samples and too small to account for the full effect. Local strain fields that are not detected in standard XRD analysis, but may well be detected by XAS, are therefore probably more important.

The limited potential of $n$ for increasing $T_{\mathrm{C}}$ of La- and Lu-doped $\mathrm{EuO}$ and the indications for a lattice compression related enhancement of $T_{\mathrm{C}}$ strengthen the importance of compressive strain in the quest to further enhance the $T_{\mathrm{C}}$ of $\mathrm{EuO}$ thin films. Up to now it is unknown if EuO films can indeed be compressively strained with sufficient thickness so that $T_{\mathrm{C}}$ can be enhanced. One reason for this is a lack of suitable substrates. Fabrication of alternative substrate materials such as $\mathrm{LuVO}_{4}(-3.4 \%$ strain for an epitaxial and commensurate film) may allow testing this approach. According to our results, the $T_{\mathrm{C}}$ enhancement should be strongest in conjunction with doping with small magnetic ions and possibly oxygen vacancies. The low-temperature, close to flux-matched deposition technique developed in this work is ideally suited to further explore these routes.

\section{ACKNOWLEDGMENTS}

The work at Cornell was supported by the National Science Foundation (NSF) [Platform for the Accelerated Realization, Analysis, and Discovery of Interface Materials (PARADIM)] under Cooperative Agreement No. DMR-1539918. This work made use of the Cornell Center for Materials Research Shared Facilities, which are supported through the NSF MRSEC program (Grant No. DMR-1719875). Substrate preparation was performed in part at the Cornell NanoScale Facility, a member of the National Nanotechnology Coordinated Infrastructure (NNCI), which is supported by the NSF (Grant No. ECCS15420819). The work in Augsburg was supported by the DFG (Grant No. TRR 80).
[1] B. T. Matthias, R. M. Bozorth, and J. H. Van Vleck, Phys. Rev. Lett. 7, 160 (1961).

[2] M. R. Oliver, J. O. Dimmock, A. L. McWhorter, and T. B. Reed, Phys. Rev. B 5, 1078 (1972).

[3] Y. Shapira, S. Foner, and T. B. Reed, Phys. Rev. B 8, 2299 (1973).

[4] K. Y. Ahn and M. W. Shafer, J. App. Phys. 41, 1260 (1970).

[5] P. G. Steeneken, L. H. Tjeng, I. Elfimov, G. A. Sawatzky, G. Ghiringhelli, N. B. Brookes, and D.-J. Huang, Phys. Rev. Lett. 88, 047201 (2002).

[6] A. Schmehl, V. Vaithyanathan, A. Herrnberger, S. Thiel, C. Richter, M. Liberati, T. Heeg, M. Röckerath, L. Fitting
Kourkoutis, S. Mühlbauer, P. Böni, D. A. Muller, Y. Barash, J. Schubert, Y. Idzerda, J. Mannhart, and D. G. Schlom, Nat. Mater. 6, 882 (2007).

[7] A. G. Swartz, Appl. Phys. Lett. 97, 112509 (2010).

[8] D. V. Averyanov, C. G. Karateeva, I. A. Karateev, A. M. Tokmachev, A. L. Vasiliev, S. I. Zolotarev, I. A. Likhachev, and V. G. Storchak, Sci. Rep. 6, 22841 (2016).

[9] C. Caspers, A. Gloskovskii, M. Gorgoi, C. Besson, M. Luysberg, K. Z. Rushchanskii, M. Ležaić, C. S. Fadley, W. Drube, and M. Müller, Sci. Rep. 6, 22912 (2016).

[10] D. V. Averyanov, I. S. Sokolov, A. M. Tokmachev, I. A. Karateev, O. A. Kondratev, A. N. Taldenkov, O. E. Parfenov, 
and V. G. Storchak, J. Magn. Magn. Mater. 459, 136 (2018).

[11] T. Mairoser, J. A. Mundy, A. Melville, D. Hodash, P. Cueva, R. Held, A. Glavic, J. U. R. Schubert, D. A. Muller, A. Schmehl, and D. G. Schlom, Nat. Commun. 6, 1 (2015).

[12] T. R. McGuire and M. W. Shafer, J. Appl. Phys. 35, 984 (1964).

[13] F. Holtzberg, T. R. McGuire, S. Methfessel, and J. C. Suits, Phys. Rev. Lett. 13, 18 (1964).

[14] A. Mauger, M. Escorne, C. Godart, J. P. Desfours, and J. C. Achard, J. Phys., Colloq. 41, C5 (1980).

[15] K. Y. Ahn and T. R. McGuire, J. Appl. Phys. 39, 5061 (1968).

[16] A. Melville, T. Mairoser, A. Schmehl, D. E. Shai, E. J. Monkman, J. W. Harter, T. Heeg, B. Holländer, J. Schubert, K. M. Shen, J. Mannhart, and D. G. Schlom, Appl. Phys. Lett. 100, 222101 (2012).

[17] S. G. Altendorf, Appl. Phys. Lett. 104, 052403 (2014).

[18] A. Mauger and C. Godart, Phys. Rep. 141, 51 (1986).

[19] N. J. C. Ingle and I. S. Elfimov, Phys. Rev. B 77, 121202 (2008).

[20] A. Melville, T. Mairoser, A. Schmehl, T. Birol, T. Heeg, B. Holländer, J. Schubert, C. J. Fennie, and D. G. Schlom, Appl. Phys. Lett. 102, 062404 (2013).

[21] A. Mauger, Phys. Status Solidi B 84, 761 (1977).

[22] T. Mairoser, A. Schmehl, A. Melville, T. Heeg, L. Canella, P. Böni, W. Zander, J. Schubert, D. E. Shai, E. J. Monkman, K. M. Shen, D. G. Schlom, and J. Mannhart, Phys. Rev. Lett. 105, 257206 (2010).

[23] J. M. Riley, F. Caruso, C. Verdi, L. B. Duffy, M. D. Watson, L. Bawden, K. Volckaert, G. van der Laan, T. Hesjedal, M. Hoesch, F. Giustino, and P. D. C. King, Nat. Commun. 9, 2305 (2018).

[24] T. Mairoser, A. Schmehl, A. Melville, T. Heeg, W. Zander, J. Schubert, D. E. Shai, E. J. Monkman, K. M. Shen, T. Z. Regier, D. G. Schlom, and J. Mannhart, Appl. Phys. Lett. 98, 102110 (2011).

[25] R. W. Ulbricht, A. Schmehl, T. Heeg, J. Schubert, and D. G. Schlom, Appl. Phys. Lett. 93, 102105 (2008).
[26] R. Sutarto, S. G. Altendorf, B. Coloru, M. Moretti Sala, T. Haupricht, C. F. Chang, Z. Hu, C. Schüßler-Langeheine, N. Hollmann, H. Kierspel, J. A. Mydosh, H. H. Hsieh, H.-J. Lin, C. T. Chen, and L. H. Tjeng, Phys. Rev. B 80, 085308 (2009).

[27] See Supplemental Material at http://link.aps.org/supplemental/ 10.1103/PhysRevMaterials.4.104412 for additional information on the deposition method of EuO thin films at low temperature, measurement of dopant incorporation, structural quality as measured by x-ray diffraction, determination of magnetic and electrical properties, comparison to previous work, and determination of the lanthanum valence in La-doped $\mathrm{EuO}$ thin films by STEM EELS.

[28] T. Mairoser, F. Loder, A. Melville, D. G. Schlom, and A. Schmehl, Phys. Rev. B 87, 014416 (2013).

[29] Landolt-Bornstein: Numerical Data and Functional Relationships in Science and Technology, edited by K.-H. Hellwege, New Series, Group III (Springer, Berlin, 1975), Vol. 7, Part b1, p. 33.

[30] R. D. Shannon, Acta Crystallogr., Sect. A 32, 751 (1976).

[31] P. M. Fahey, P. B. Griffin, and J. D. Plummer, Rev. Mod. Phys. 61, 289 (1989).

[32] P. M. Voyles, D. A. Muller, J. L. Grazul, P. H. Citrin, and H.-J. L. Gossmann, Nature 416, 826 (2002).

[33] J. A. Mundy, D. Hodash, A. Melville, R. Held, T. Mairoser, D. A. Muller, L. Fitting Kourkoutis, A. Schmehl, and D. G. Schlom, Appl. Phys. Lett. 104, 091601 (2014).

[34] D. C. Mueller and W. Fichtner, Phys. Rev. B 70, 245207 (2004).

[35] D. V. Averyanov, O. E. Parfenov, A. M. Tokmachev, I. A. Karateev, O. A. Kondratev, A. N. Taldenkov, M. S. Platunov, F. Wilhelm, A. Rogalev, and V. G. Storchak, Nanotechnology 29, 195706 (2018).

[36] S. G. Altendorf, N. Hollmann, R. Sutarto, C. Caspers, R. C. Wicks, Y.-Y. Chin, Z. Hu, H. Kierspel, I. S. Elfimov, H. H. Hsieh, H.-J. Lin, C. T. Chen, and L. H. Tjeng, Phys. Rev. B 85, 081201(R) (2012). 